\title{
Organic Underlayers for EUV Lithography
}

\author{
Douglas J. Guerrero, ' Carol Beaman,' \\ Rikimaru Sakamoto, ${ }^{2}$ Takafumi Endo, ${ }^{2}$ Bang-Ching $\mathrm{Ho}^{2}$ \\ ${ }^{I}$ Brewer Science, Inc. \\ Rolla, MO 65401, USA \\ ${ }^{2}$ Nissan Chemical Industries, Electronic Materials Research Laboratories \\ Toyama 939-2792, Japan \\ dguerrero@brewerscience.com
}

\begin{abstract}
The density, absorbance, and outgassing of underlayer films and the effect of EUV irradiation on such films were evaluated. The relationship between density and calculated EUV absorbance was determined. Films having lower film density have lower EUV absorbance, but this phenomena can be offset by adding a halogen into the polymer matrix. Most materials have low outgassing during EUV exposure when compared to industry standard targets. Surface analysis using contact angle and optical characterization showed good stability of underlayer films under EUV irradiation.
\end{abstract}

Keywords: underlayer, outgassing, EUV

\section{Introduction}

As device generations continue towards the 22-nm node, EUV lithography is expected to be the method of choice in order to achieve the required $\mathrm{CD}$ targets. One of the main challenges in material design, particularly with resists, is the improvements in line width roughness (LWR) and sensitivity needed to achieve industry targets. The ITRS roadmap ${ }^{1}$ has set targets for $3 \sigma$ LWR at $<3 \mathrm{~nm}$ and for sensitivity at $<10 \mathrm{~mJ} / \mathrm{cm}^{2}$. Most resists to date do not meet both targets, and there seems to be a compromise between LWR and sensitivity. There have been several publications that have reported methods to try to improve resist sensitivity while improving LWR. Among the various approaches are incorporating PAG functionality ${ }^{2,3}$ or halogens ${ }^{4,5}$ into the resist polymer, adding conducting polymer layers beneath the resist $^{6}$, or using PAG containing monolayers prior to resist application. ${ }^{7,8}$ Nevertheless a comprehensive study including all factors that affect underlayer performance has not been presented. It has been suggested that resist absorbance ${ }^{9}$ and secondary electron generation by EUV irradiation play an important role in acid generation that can improve LWR and sensitivity. ${ }^{10}$

In conventional lithography ( $>157 \mathrm{~nm})$, the resist and underlayer (bottom anti-reflective coating) have two distinct and functional optical parameters (refractive index and absorbance). However, under EUV energy, resist and underlayer behave as a single layer. There are no reflection effects, and both materials absorb approximately the same amount. Our approach is to use the high-energy EUV photon, which is about 14 times the energy of 193-nm wavelength, and secondary electron generation to enhance the resist acid generation efficiency and therefore improve LWR and sensitivity. The concept is illustrated in Scheme 1. 


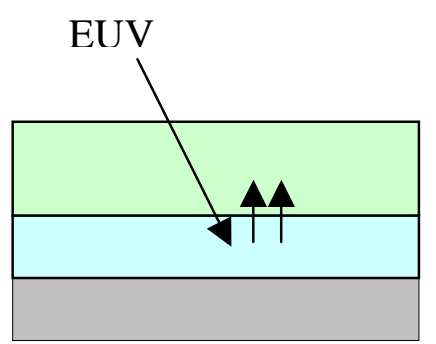

Scheme 1. Concept illustrating secondary electron effect on acid generation in resist.

In this paper we investigated the relationship between material density, absorbance, and structure as a means to influence secondary electron generation. In addition, another major concern for EUV lithography is the potential outgassing that can occur upon EUV exposure. Outgassed materials can coat the reflective mirrors and decrease exposure energy on the substrate. Another objective of this paper was to find the relationship between structure and material outgassed during EUV exposure. Finally, we tested the effect of underlayers on the performance of EUV resist. The goal was to determine the relationship between underlayer absorbance and its effect on resist LWR and sensitivity.

\section{Method}

\subsection{Materials and process}

The materials used were selected from our library of anti-reflective coatings. These materials represent a wide variety of functional groups and structure types. The base resins were formulated with crosslinker, catalyst, and surface enhancing additives in common resist solvents. The materials are classified as follows:

Table 1. Chemical platforms studied.

\begin{tabular}{|c|c|}
\hline Platform & Underlayers \\
\hline Methacrylates & D, F, H \\
\hline Silicon containing & A, E, G \\
\hline Novolacs & B, C \\
\hline Linear polyesters & J, K \\
\hline Branched polyesters & I, M \\
\hline
\end{tabular}

All films were coated to a thickness of $20 \mathrm{~nm}$ on silicon substrates and baked at $205^{\circ} \mathrm{C}$ for 60 seconds.

\subsection{Outgassing measurements}

Outgassing tests were done using the Resist Outgassing Exposure system (ROX) at the College of Nanoscale Science and Engineering (CNSE) at State University of New York at Albany. Pieces of coated wafers were placed in a vacuum chamber and irradiated $\left(15 \mathrm{~mJ} / \mathrm{cm}^{2}\right)$ with an Energetiq EQ10M 10W EUV source. The evolving gases were trapped and analyzed using a quartz crystal microbalance (QCM) and quadrupole mass spectrometer.

\subsection{Contact angle measurements}

Water contact angle measurements were taken to determine surface changes on the films before and after EUV exposure. The measurements were done in an AST Products VCA Optima system under static conditions.

\subsection{Absorbance determination}

Film absorbance at EUV wavelength $(13.5 \mathrm{~nm})$ was calculated using the method developed by the Center for X-ray Optics at Lawrence Berkeley National Laboratory. ${ }^{11}$ This method requires the input of molecular formula composition and film density values.

\subsection{Density measurements}

Density measurements were carried out by X-ray reflectivity techniques at the Missouri University of Science at Technology (MST) using a PANalytical X'Pert Materials Research Diffractometer with a $\mathrm{Cu}$ tube. The incident beam optics module consisted of an X-ray mirror, and the diffracted beam optics module was a 0.18-degree parallel plate collimator.

\subsection{Optical measurements}

Optical changes before and after EUV exposure were determined using spectroscopic ellipsometry in a J.A. Woollam M-2000 ${ }^{\circledR}$ system.

\section{Results and Discussion}

\subsection{Film density}

Before the underlayer EUV absorbance can be calculated, the film density must first be determined. Film density values were extracted from the interference fringes obtained and previously published procedures. $^{12}$ All of the coated films gave good interference fringes (Figure 1) except for sample K (Figure 2). The lack of interference fringes was attributed to the film having high 
surface roughness causing reflectivity to become more diffused and less specular. We were not able to calculate a density value for sample $\mathrm{K}$.

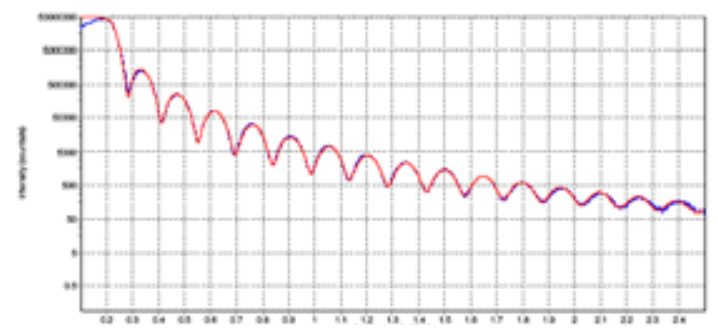

Figure 1. Example of interference fringes obtained for all underlayers, except underlayer $\mathrm{K}$.

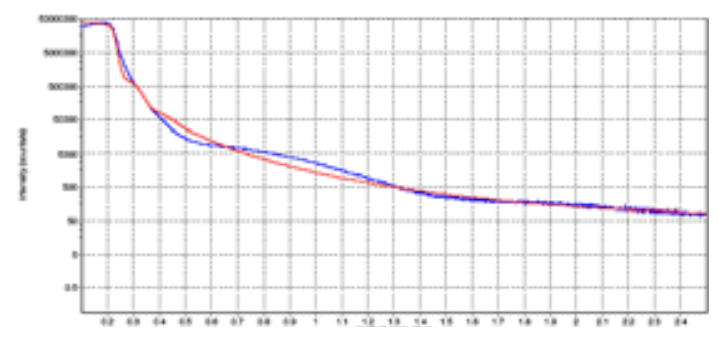

Figure 2. No interference fringes obtained for underlayer $\mathrm{K}$.

All data were fitted best using a two-layer model, meaning that the films exhibit a density gradient from top to bottom. For most films, the density on the top 5-6 nm was higher than in the bulk of the film. The change in film density through film thickness has been observed before by us ${ }^{13}$ and others ${ }^{14}$ and is not totally unexpected. When thin films are spin coated and baked, a redistribution of components can occur, causing different crosslink densities throughout the film. The bulk density for all samples ranged from $0.76-1.29 \mathrm{~g} / \mathrm{cm}^{3}$ (Figure $3)$.

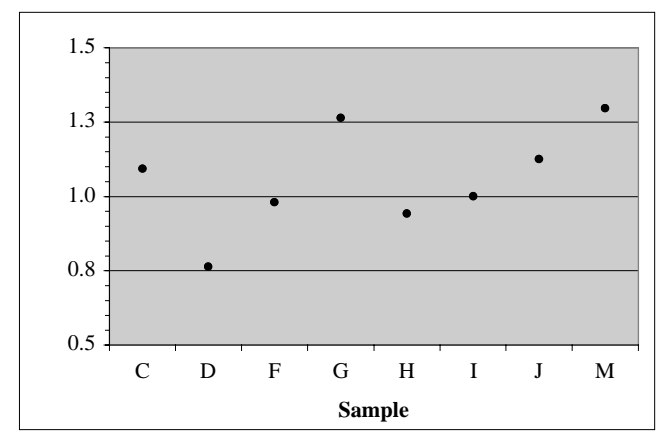

Figure 3. Bulk film densities for underlayers tested.
There was not a strong correlation between density values and structure types; however, the methacrylate polymers $(\mathrm{D}, \mathrm{F}, \mathrm{H})$ gave the lowest density values, probably due to less rigid nature of these polymers. The absorbances of the underlayers were calculated using the bulk density values.

\subsection{Film absorbance}

Absorbance values were calculated from the density values obtained above. The relationship between density and absorbance is shown in Figure 4. As the density increases, the absorbance increases. However, the ability to increase absorbance by using density changes alone is limited to the 3-5 absorbance units per micron range. Most organic materials, including some experimental EUV resists, ${ }^{9}$ have shown similar absorbance values. Underlayer $\mathrm{M}$ deviated from this trend. Underlayers $\mathrm{M}$ and I are structurally similar with the only difference being that film $\mathrm{M}$ contains a halogen substituting some of the hydrogen atoms. The effect of adding a halogen to the polymer backbone has a significant effect in increasing absorbance. Similar trends have been observed with resists. ${ }^{4}$

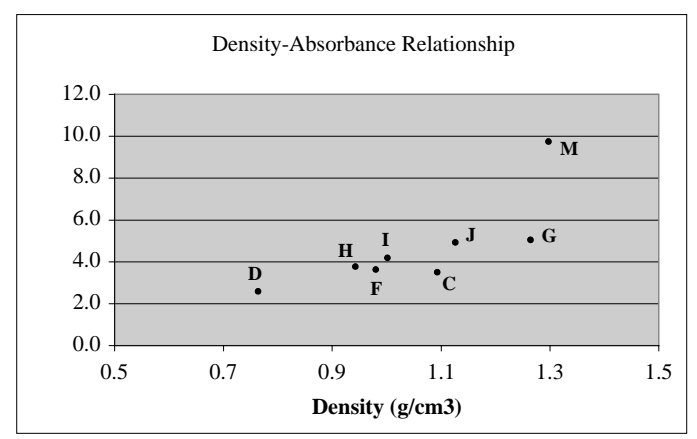

Figure 4. Relationship between density and calculated absorbance at EUV wavelength.

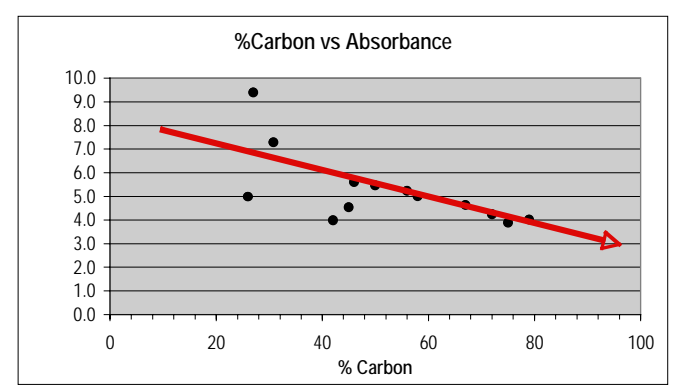

Figure 5. Relationship between \% carbon content and absorbance. 
In addition to halogen addition, we determined the effect of carbon content on absorbance. We found that the amount of \% carbon increases as absorbance decreases. A similar relationship and the benefits of having low carbon content polymers for EUV lithography have been reported in the literature. ${ }^{5}$

\subsection{Outgassing during EUV exposure}

Reducing or eliminating material outgassing from resist films during EUV irradiation is desirable to avoid contamination of the reflective lens system inside the EUV exposure tool. The results from the outgassing tests are shown in Figure 6. The total outgassed material (molecules $/ \mathrm{cm}^{2}$ ) for all materials was lower than the target established by International SEMATECH recommendations.

We also looked at the effect of outgassing when using the combined underlayer-resist stack. The results show that the amount of outgassing observed using the underlayer alone is not added to the resist amount. In fact, total outgassing of the underlayer-resist stack is the same at that for the resist alone. Therefore, the contribution to total outgassing from the underlayer is minimal compared to resist amounts.

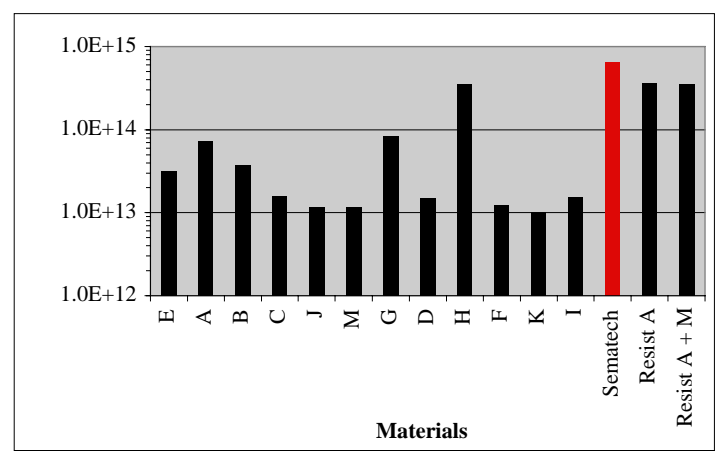

Figure 6. Outgassing amount for underlayers tested during EUV exposure.

The fragmentation patterns of the outgassed species revealed that the simplest methacrylate structure (underlayer $\mathrm{H}$ ) is more likely to outgas than similar structures having more aromatic content (underlayers D, F). Among the siliconcontaining materials (underlayers A, E, G), the fragmentation analysis did not show siliconcontaining species, rather fragments coming from structures attached to the silicon atom. Stability of the silicon-containing polymers is a function of the pendant functional groups attached to the silicon atom. The highly aromatic series (underlayers B, C) show low outgassing, with slightly less stability for the system having aliphatic groups attached to the aromatic backbone (underlayer $\mathrm{B}$ ). The polyester series, either branched or linear, performed very well under these test conditions.

Another important feature of merit is the rate of outgassing (molecules $/ \mathrm{cm}^{2}$ per second of exposure). Figure 7 compares the rate of outgassing for underlayers $\mathrm{M}$ and I, and M-resist stack. When the rates are compared, the resist and underlayer $\mathrm{M}$ resist stack are significantly higher and slightly below the established targets.

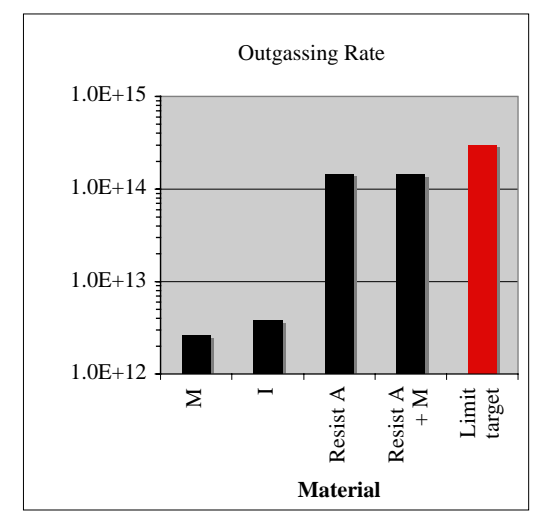

Figure 7. Outgassing rate for underlayers $\mathrm{M}$ and $\mathrm{I}$ and resist.

3.4 Effect of EUV irradiation on underlayer surface In addition to outgassing, we wanted to determine the damaging effect of EUV exposure on materials by using contact angle measurements. The data were summarized in Figure 8 below. The outgassing data do not necessarily correlate with the contact angle changes observed. That is, the highest outgassing underlayer did not have the most change in contact angle. This is probably due to the fact that while the contact angle technique is able to detect surface modifications, the outgassing test measures material coming from the bulk of the film. In Figure 8, changes $<2$ degrees are assumed to be insignificant. The most change occurred with underlayers B and E. Underlayer B became more hydrophobic while $\mathrm{E}$ became more hydrophilic. Underlayer B outgassing data showed lost polar fragments attached to aromatic backbone, thus making the polymer more hydrophobic. On the other hand, for the silicon-containing underlayer E, 
loss of aliphatic fragments during EUV exposure caused it to be more hydrophilic (due to possible formation of $\mathrm{SiOH}$ on the surface).

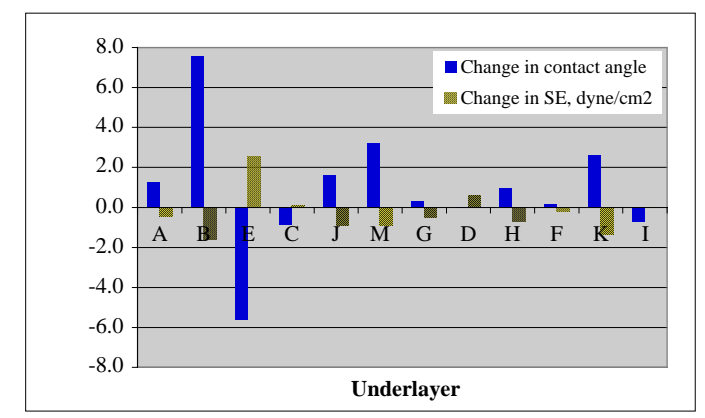

Figure 8. Contact angle and surface energy changes for undelayers after EUV exposure.

\subsection{Effect of EUV irradiation on optical properties}

Most of these underlayers are derived from antireflective coatings for ArF and $\mathrm{KrF}$ lithography. As such, the materials contain light-absorbing moieties for these wavelengths. These optical tags allowed us to follow the structural changes of the underlayers during EUV exposure. Absorbance spectra in the 190- to 400-nm range were generated after coating the films, after placing the films under high vacuum, and after exposure at 15 and $120 \mathrm{~mJ} / \mathrm{cm}^{2}$. The extremely high dose provided us with the worst possible case for damaging the underlayers.

The results show that most underlayers were very stable under the test conditions described. Underlayers with the greatest changes observed were those containing $\mathrm{KrF}$ chromophores likely due to photobleaching effects that are also possible under normal light exposure.

\subsection{Lithographic evaluations}

At the time of this printing, we were not able to provide lithographic performance of the various underlayers under study. These studies are underway and expect to be published at a later date.

\section{Conclusions}

A series of materials were developed for use as underlayers for EUV lithography. It was shown that through compositional changes the density and absorbance of the underlayers can be modulated. However, the biggest impact in increasing underlayer absorbance is the addition of a halogen to the composition. Addition of a silicon- containing polymer did not seem to have an effect. Low carbon content also contributes to increasing absorbance, which makes simple methacrylate polymers attractive for this use, but with the risk of being unstable under EUV exposure conditions. The underlayer with most outgassing in this study was a simple methacrylate polymer. At the time of this writing, the greatest concern for outgassing seems to come from resist. Contact angle and optical properties analysis of underlayers before and after EUV irradiation at extremely high doses revealed fairly stable films. Lithographic evaluations are currently under evaluation.

\section{Acknowledgments}

The authors would like to thank the useful discussions of the various test facilities during the course of this research.

\section{References}

1. www.itrs.net/Links/2007ITRS/2007_Chapters/ 2007_Lithography.pdf

2. W. Yueh, US Patent, 7,005,277 B2 (2004).

3. M. Wang, C-T. Lee, C. L. Henderson, W. Yueh, J. M. Roberts, K. E. Gonsalves, Proc. SPIE, 6923 (2008), 6923-40 in press.

4. T. Sasaki, O. Yokokoji, T. Watanabe, H. Kinishita, Proc. SPIE, 6923, (2008) 6923-166, in press. And references therein.

5. T. Fedynyshyn, R. B. Goodman, J. Roberts, Proc. SPIE, 6923, (2008), 6923-47, in press.

6. R. P. Meagley, US Patent, 2006/0003253 A1 (2006).

7. R. P. Meagley, J. of Macromolecular Sci., Part: B, Physics, 46, (2007), 33-41.

8. S. Sharma, R. P. Meagley, Proc. SPIE, 6519, (2007), 65190P.

9. M. Chandhok, H. Cao, W. Yueh, E. M. Gullikson, R. L. Brainard, S. A. Robertson, Proc. SPIE, 5374, (2004), 861.

10. R. Brainard et al., Proc. SPIE, 6923, (2008), 6923-84, in press.

11. //henke.lbl.gov/optical_constants/getdb2.html

12. T. C. Huang, R. Giller, G. Will, Thin Solid Films, 230 (2), (1993), 99-101.

13. Unpublished results using neutron scattering techniques.

14. J. Algers, R. Suzuki, T. Ohdaira, F. H. J. Maurer, Polymer, 45 (13), (2004), 4533-4539. 